\title{
Algunos valores intangibles de la mediación como estrategia de marketing
}

\author{
Some mediation's intangible value as marketing strategy
}

Recibido: 18-04-2021 | Aceptado: 08-07-2021

Vanny Maltez Navarro*

*https://orcid.org/0000-0002-7587-7881

Nicaragua

\section{Resumen}

El presente artículo académico describe cómo los valores intangibles de la mediación son utilizados en estrategias de mercadeo y de qué forma favorecen el consumo del servicio de los métodos de solución de conflictos.

Se presenta un acercamiento teórico del valor intangible de los servicios desde el enfoque del marketing, describiendo ciertos valores intangibles de la mediación que favorecen el su posicionamiento como opción para resolver un conflicto. Presentando los intangibles de competitividad, la innovación, la modernidad, el empoderamiento y la innovación cómo materias de estudio necesarias en la finalidad de comprender la interacción de consumo existente entre los valores intangibles, la percepción y el posicionamiento.

De igual manera se realiza un acercamiento teórico de cómo el consumidor interpreta estos valores intangibles de la mediación en su esquema mental, para tomar decisiones futuras y extender recomendaciones a otros individuos de la sociedad, dependiendo del grado de expectativa y satisfacción obtenido en el proceso de percepción; con la finalidad de logar un posicionamiento en la mente del consumidor.

Se describe, desde la visión del marketing, el posicionamiento de la mediación como resultado de la estrategia de los valores intangibles. Para ello se ponen en discusión la teoría del comportamiento del consumidor en términos de expectativa, satisfacción y los posibles estadios de resultados para el posicionamiento de la mediación.

Cómo citar

Maltez-Navarro, V. Algunos Valores Intangibles De La

Mediación Como Estrategia De Marketing. MSC Métodos

De Solución De Conflictos. Recuperado a partir de https://

revistamsc.uanl.mx/index.php/m/article/view/1 
Para realizar conclusiones descriptivas de cómo los valores intangibles de la mediación benefician el consumo por medio de una mejora en el posicionamiento en la mente del consumidor.

Palabras clave: Mediación, Valores Intangibles, Estrategia, Percepción, Posicionamiento.

\begin{abstract}
This study analyzed the relationship between interpersonal conflict management styles (aggressive, passive and cooperative) among adolescent schoolmates and a set of psychosocial skills (emotional intelligence and multidimensional empathy). The participants were 125 adolescent middle-school students belonging to a public school in Guadalajara, Jalisco, Mexico. They answered the Conflictalk Questionnaire, the Trait Meta-mood Scale short version, and the Interpersonal Reactivity Index. The findings showed statistically significant positive correlations between the cooperative style and the aforementioned skills, and gender differences. The implications of these findings are discussed in the context of the literature on school coexistence, peace education and interpersonal conflict management in adolescents.
\end{abstract}

Key words: Mediation, Intangible Values, Strategy, Perception, Positioning.

\section{INTRODUCCIÓN}

El presente artículo aborda la perspectiva de la teoría del comportamiento del consumidor del marketing (Schiffman \& Kanuk, 2010) y la teoría de los valores intangibles de la medición (Gorjón, 2017), presentando una discusión entre ambas teorías, los distintos enfoques teóricos del valor intangible, en ciencias económicas y administrativas, y de qué forma se acopla con los valores intangibles de la competitividad, la innovación, la modernidad, el empoderamiento y la especialización aplicados en la mediación. Para presentar elementos de estudio de la percepción (Armstrong \& Kotler, 2013, p. 82) para el posicionamiento (Ampuero \& Vila, 2006, p. 102) en la mente de los consumidores, por medio de la estrategia de mercadeo con base a los valores intangibles. Enfocándose en variables como la expectativa, la satisfacción y el posicionamiento.

Como objetivo el artículo pretende converger los valores intangibles de la mediación y las variables de la percepción y su proceso de selección, organización e interpretación. Enfatizando la relevancia de los valores intangibles como objeto de estudio, para lograr un satisfactorio cumplimiento de las necesidades y un posicionamiento adecuado de la mediación en el consumidor.

El desarrollo del artículo cuenta con la identificación de ciertos valores intangibles que benefician a la mediación y su finalidad, centrando su atención en que intervienen en las expectativas y satisfacción de la per- 
cepción del consumidor para obtener como resultado uno de los distintos estadios del posicionamiento.

Concluyendo con una reflexión de relevancia científica que representa la percepción de los valores intangibles en cuanto al interés de los individuos obtenido mediante la solución del conflicto a través de la mediación.

\section{EL VALOR INTANGIBLE EN LOS SERVICIOS DESDE LA PERSPECTIVA DEL MARKETING}

El concepto de valor, visto desde una perspectiva del marketing, se establece en nociones asociadas a la percepción de un sujeto en cuanto a la utilidad, beneficios y calidad obtenida por un servicio pagado (Gil Saura \& González Gallarza, 2008, p. 13). La postura del consumidor al hacer uso de un servicio se centra en evaluar el esfuerzo psicológico y material otorgado en contraposición con el beneficio o utilidad obtenida por haber utilizado ese servicio.

A diferencia de los productos, que normalmente se encuentran en el mercado, los servicios como la mediación presentan una complejidad para poder transmitir los beneficios, calidad y utilidad obtenida. Esto debido a no presentar la experiencia que ofrece un producto físico por carecer de sensaciones. Por ello es que los servicios deben de ser capaces de transformar estos beneficios en una experiencia y, al mismo tiempo, resaltar estos beneficios a los consumidores de servicios.
Ortega y Gasset (1991, pág. 29) describen que el valor se obtiene de un proceso cognitivo, a la vez subjetivo, que toma lugar en la mente del consumidor con la finalidad de dar sentido a su realidad objetiva, identificando así los aspectos valioso y no valioso. De esa forma se observa que el valor es un factor perceptible resultado de una experiencia vivida por el consumidor con dos posibles resultados: positivo y negativo.

Para Schiffman y Kanuk (2010, pág. 10) el valor presenta una ecuación matemática resultante de la diferencia entre los recursos no tangibles y tangibles entregados al servicio, y los beneficios percibidos del mismo. Para los servicios el intangible se vuelve la clave de percepción de valor en la experiencia que tenga el consumidor en el servicio, estos beneficios son transmitidos mediante reafirmaciones sensoriales en el individuo.

Todos estos valores intangibles no son reconocidos por el consumidor hasta pasar por su experiencia, sin embargo, la información de la oferta existente en el ambiente genera en el consumidor una noción de lo que podría resultar por adquirir un producto o experimentar un servicio, conocido en la teoría del comportamiento del consumidor como expectativa.

Al igual que el valor generado en un servicio, la evaluación de la expectativa se calcula en una ecuación neta de valor: valores positivos menos valores negativos percibidos por el consumidor. Aplicados a la mediación los valores negativos se reflejan en la sensación de pérdida emocional, material y temporal; y los valores positivos son re- 
presentados en la medición de los valores intangibles de la mediación vistos desde un enfoque de provecho teórico en las ciencias económicas y administrativas. Los valores intangibles presentados en el presente artículo tienen la característica de potenciar el posicionamiento de la oferta de un servicio. Estos valores intangibles son: la competitividad, la innovación, la modernidad, el empoderamiento y la especialización.

\section{EL INTANGIBLE DE LA COMPETITIVIDAD}

La competitividad interviene en todas las actividades diarias del ser humano, en el proceso de tomar una decisión los consumidores se encuentran bombardeados de múltiples estímulos que afectan su capacidad racional y emocional de decisión. Estos estímulos son provenientes no solamente de un objeto, que puede ser un producto, sino que detrás de este objetivo existe todo un trabajo enfocado y diseñado por el marketing para influenciar la decisión de una persona. Por lo que hace al primer punto de importancia de los valores intangibles y la percepción de los mismos por los consumidores, la percepción del imaginario social les permite a los operadores de distintas ciencias utilizarlos como diferenciación, por medio del cual los operadores ofrecen mayores estándares de calidad y un mayor grado de satisfacción, algo determinante para el éxito de las organizaciones.

Así como en las ciencias económicas el capital humano y el capital estructural forman parte de una cadena productiva generado- ra de competitividad, así es para los operadores de los Métodos de Solución de Conflictos, siendo estos el capital intelectual. De esta manera lo expone López, Nevado, y Baños (2008, pág. 49) sobre la relevancia del capital intelectual que trasciende el mero ámbito empresarial, ya que está vinculado directamente al crecimiento económico, la competitividad y el propio desarrollo. Formando un centro de eje competitivo las cualidades, características, habilidades y conocimiento (Gorjón, 2017, p. 79) presentes en la persona para brindar soluciones a los problemas de los individuos. Esto genera una correlación entre el crecimiento personal y el éxito de una organización. Esto se traduce en que una persona más capacitada para brindar soluciones a otra con una necesidad es más productiva y proporcionar así satisfacción a los consumidores, generando como resultado la competitividad de la organización.

Unger, Flores e Ibarra (2014, p. 936) exponen que los trabajadores productivos, porque tienen todas las herramientas conceptuales y experienciales, son más productivos para las organizaciones y en contra remuneración reciben un mayor salario por su productividad, siendo así la representación de competitividad organizacional y competitividad personal.

Por ello la competitividad es un valor intangible que será potenciado con la investigación, los operadores de los MSC podrán conocer cuáles son los atributos intangibles determinantes al momento de tomar la decisión por parte de los usuarios de los MSC, de esta forma el operador 
puede ofrecer justamente lo que el usuario necesita.

Incrementando su eficacia como operador, podrá así mismo incrementar el alcance de los MSC hacia la población, mejorando la productividad, el desarrollo tanto personal como del servicio de la mediación, generando una competitividad marcada de los MSC entre las distintas formas de solucionar las controversias y así mismo desarrollando una competitividad en el capital humano, y con ello más valor en ambas vías.

La investigación brindará también herramientas conceptuales al operador de los MSC y podrá así proveer más valor a su Know-How, a su experticia y a la profesión le dotar de un marco conceptual riguroso de cómo debe ofrecerse un MSC con la finalidad de diferenciarse competitivamente del sistema de justicia tradicional.

\section{EL INTANGIBLE DE LA INNOVACIÓN}

Uno de los factores existentes siempre en las nuevas formas de pensar, nuevos procesos, servicios y desarrollos de productos es la innovación. Torres (2016, p: 117) refiere a la existencia de una cultura de innovación cuando un conjunto de individuos, descritos como comunidad, tienen la posibilidad de elaborar y ofrecer productos, servicios, procesos con un grado de calidad y competitividad ante los demás, por medio de sus conocimientos, experiencias y habilidades adquiridas.

La innovación de productos, servicios y procesos depende de nuevas adecuaciones que faciliten la solución de una necesidad y a su vez genere mayor calidad a los servicios actuales. Para las ciencias económicas según Drucker (1988) la innovación debe de constar con un análisis de las oportunidades, informarse de los consumidores, aplicación, pequeñas escalas y liderazgo (Godoy, 2012). El concepto de innovación y sus criterios anteriormente descritos son aplicables a las demás prácticas sociales e investigativas, siendo así actualmente un estandarte de crecimiento organizacional, liderazgo y generador de valor. También es el caso para los MSC, en donde el resultado de la investigación cumplirá con un exhaustivo análisis de las oportunidades para desarrollar los MSC con información directamente de los usuarios, como distintivo generador de valor. La innovación se ha vuelto un tema tan relevante, no sólo a nivel empresarial, sino en distintas organizaciones y debido a los altos grados de competencia existente en la sociedad, la innovación es clave para poder conseguir el éxito. Como bien se ha expresado anteriormente la innovación no únicamente reside en ofrecer un producto $o$ un servicio nuevos, sino en la capacidad de las personas de transformar los procesos de una forma más eficiente (Moraleda, 2004, pág. 136).

Con la transformación de los procesos se provoca una disrupción en la forma que se brinda soluciones a la sociedad. Aplicado a los MSC, la actual forma de ofrecer el servicio se transforma con nuevas ideas, otros enfoques y valores, con otra perspectiva para aportar a la eficiencia y eficacia; y así convertir las actuales maneras de realizar las cosas en discursos desfasados. 
A este proceso de sustitución de los procesos, por otros más eficientes y eficaces, como resultado de la innovación Schumpeter le denomina destrucción creativa y lo considera una expansión cíclica, es decir, siempre van a estar presente en la sociedad, (González Ulloa Aguirre, 2014, pág. 136).

Por esta razón el intangible de la innovación es importante en la investigación, los nuevos conocimientos potencian y modifican los procesos y con ello los operadores de los MSC puedan modificar su discurso, parte operativa de los MSC, para hacer ver a la población de forma clara los beneficios de los MSC y así incrementar el alcance de los mismos.

Así mismo contribuirá al fortalecimiento de la profesión de los MSC, innovando los procedimientos operativos y las distintas formas de ofrecer el servicio según la segmentación y el conflicto que enfrentarán podrá ser manejado, transformando los procesos y los modelos actuales a figuras más eficientes y eficaces.

\section{EL INTANGIBLE DE LA MODERNIDAD}

La modernidad se caracteriza por el avance hacia adelante y hacia arriba, sin embargo, tiene características temporales y espaciales. Esto significa que la modernidad es parte de un proceso de mejora continua en donde los valores van cambiando su perspectiva y la forma en que se enfocan, con criterios propios de un grupo de personas en geografías específicas y temporalidad determinada.
Las peculiaridades propias de un grupo de personas se encuentran directamente relacionadas a la aceptación del consenso social entre lo moralmente aceptable, lo no aceptable, lo nuevo y lo antiguo. Estos agentes se pueden entender como el tejido cultural que envuelve a una determinada sociedad, y donde la temporalidad y el territorio delimita los atributos de la modernidad propios de una sociedad (Romero, 2002, pág. 15).

Esta característica de progreso lineal hacia adelanta, denominada modernidad, está en todas las formas sociales y no solamente en la visión tecnológica, sino también en la modernidad de procesos, modernidad de información y modernidad en la forma de dar solución a una necesidad (Álvarez, 2015, pág. 56). Es así entonces, que los MSC son una respuesta a la necesidad de brindar justicia de una forma moderna, y en concordancia a eso sus procesos de información deben también actualizarse permanentemente.

Como progreso a la modernidad doctrinaria la investigación propone un paso hacia adelante enfocada en cambiar la forma de ver el valor de los MSC y la forma distintiva en que los operadores deben de enfocar el discurso social para su utilización. Con una propuesta de modernización del proceso operativo de los MSC, renovando el conjunto de conocimientos de los operadores hacia una nueva perspectiva con mayor impacto de persuasión.

Debido a que la modernidad es un efecto social, actúa de forma no controlada, es indispensable que el conocimiento de la 
ciencia de igual manera se modernice, los MSC no están exentos de esta necesidad. La transformación creciente de productos y servicios, con la modernización de las exigencias de la sociedad propone un campo para los MSC de adecuarse o perecer en el intento.

La nivelación de la ciencia de los MSC con las necesidades de la sociedad busca el proceso de aprendizaje y adaptación de toda ciencia a los efectos de la globalización, siendo esta una herramienta de calidad y diferenciación, y cómo expone Ávalos Rodríguez (2017, pág. 159) el cambio del paradigma del aprendizaje requiere de herramientas que permitan materializarlo y conseguir una modernización real.

Al mismo tiempo facilita la adaptación en el proceso de modernización, desde el punto de vista de los usuarios, al ser más fácil de entender, siendo más atractivos y adecuando así mismo los MSC a las exigencias actuales de justicia. Estableciendo así una delimitación espacio temporal en la forma de solucionar los conflictos de las personas, más cercano a la gente.

Por eso el intangible de la modernidad es importante en la investigación, los nuevos conocimientos modernizan los procesos de los MSC, procura aportar al alineamiento social de los MSC con la contemporaneidad de las necesidades de la sociedad, poder crecer con calidad como profesión.

\section{EL INTANGIBLE DEL EMPODERAMIENTO}

Si bien es cierto que el empoderamiento de los intangibles de la mediación están orientados a darle valor a las partes como eje fundamental del procedimiento, el término empoderamiento trasciende más allá. Por su carácter multidisciplinar y ser un concepto intangible cuya fuente de existencia es el propio ser. Es decir, no puede hablarse de empoderamiento sino se habla de las cualidades humanas y de cómo éstas las demuestra en su entorno de una forma lingüística.

Al ser el operador el responsable de llevar a cabo los procedimientos de los MSC es fácil de igual forma concluir que el empoderamiento es parte intrínseca del operador, y no únicamente de los usuarios de los MSC. El empoderamiento en la investigación tiene beneficios en ambas partes, ya que los usuarios van a tener una información más clara y detallada para su toma de decisiones, que es el fin primordial del empoderamiento.

La Organización Mundial de la Salud (OMS) define el empoderamiento como un cambio conductual en donde las personas toman control sobre sus decisiones (Domínguez-Cruz, Pereyra-Rodríguez, \& Ruiz-Villaverde, 2018, pág. 2). No obstante, es importante aclarar que esta decisión debe ser tomada con toda la información necesaria, y aquí es donde toma importancia el papel del operador. Tener un operador debidamente instruido y con todos los elementos cognitivos le permite una mejor instrucción para la toma de decisión del usuario. 
Es entonces cuando toma importancia la investigación, que tendrá como objetivo brindar a los operadores todos los valores propios de los MSC, y de esta forma transmitirlos a los usuarios para que ellos puedan tomar el control de sus decisiones. Desarrollando tanto al usuario de los MSC como al operador de los mismos, con la finalidad de incrementar sus capacidades de forma progresiva y del potencial que tienen ambos, cada quién en su rol, para la toma de decisiones.

Así lo expresa Sánchez-Vidal (2017, pág. 162) al determinar que el poder es fuente de fortaleza y capacidad humana, que permite forjar a la persona misma. Este desarrollo es de carácter progresivo e intencionado del potencial y capacidades en su interacción personal y social.

Un adecuado empoderamiento del operador en el rol que debe jugar no sólo debe de ser el conferido ante las partes al momento de acordar llevar un proceso de solución de conflictos, sino también como experto de la profesión debe de conocer todos los beneficios según el segmento. El operador de los MSC debe de estar empoderado desde el inicio del proceso y no limitarse a empoderar a las partes, ya que existe una dimensión de empoderamiento a nivel psicológico.

Caicedo Muñoz (2015, pág. 1600) propone al empoderamiento como un proceso en el tiempo el cual consta de tres niveles: el psicológico, el organizacional y el comunitario. Siendo el primero el que brinda la facultad al ser, de control y potenciación individual. Por eso el intangible empoderamiento es im- portante en la investigación, los nuevos conocimientos pretenden facilitar e instruir con mayor contundencia y claridad a los operadores y a los usuarios de los MSC con la finalidad de tomar la decisión entre los MSC y el sistema de justicia tradicional.

\section{EL INTANGIBLE DE LA ESPECIALIZACIÓN}

Parte importante de toda profesión es su grado de especialización y como gestiona la información para poder brindar soluciones a la sociedad. Al ser la especialización un intangible, inherente al ser mismo, la forma que puede el operador hacer notar su especialización es a través del lenguaje. Expresiones lingüísticas que le permitan al operador transmitir todo su bagaje de la materia en palabras simples pero contundentes y precisas.

Para poder transmitir una idea de forma coherente, mediante la comunicación efectiva es determinante saber lo que el público piensa y espera. Según Urbina-Medina (2016, pág. 115) la comunicación en temas sensibles como la salud consta de cinco principios propuestos por la (OMS), siendo el quinto tomar en consideración a la población.

Actualmente se conoce que al público usuario de los MSC no le resulta interesante el valor que estos puedan tener, sin embargo, no se tiene un marco referencial de lo que esperan. La investigación brindará, además de una mejora significativa del discurso social, una idea de las características comportamentales de los distintos segmentos de usuarios de los MSC. 
El conocer lo que le espera a la población ante un conflicto permite al operador de los MSC potenciar sus técnicas de persuasión, los éxitos de la gestión de las actividades directivas dependen a gran escala de la habilidad que tenga el operador para poder convencer e influenciar a las personas para la utilización de los MSC.

Autores como Corts, Munduate, y Medina (2008, pág. 369) proponen nueve tipologías de persuasión: racional, consulta, recurrir a las aspiraciones, adulación, recurrir a las relaciones personales, intercambio, coalición, legitimación y presión. En este sentido el operador de los MSC al estar debidamente informado de las expectativas del usuario puede aplicar técnicas relacionales, recurrir a aspiraciones, de legitimación y de persuasión racional.

De igual forma el conocer las características de la persona que va a pasar por un MSC, el operador puede utilizar su conocimiento para generar valor a lo que el usuario está por pasar, brindándole un motivo por el cual su decisión debe de ser optar por un MSC. Para lograr esto es imprescindible que el operador cuente con la especialización propia de su profesión y formación, la información y la fundamentación lógica de intelectual de los valores, de esta forma podrá conectar al usuario a los valores intangibles de los MSC.

Según González (2000, pág. 309) la interpretación del concepto valor que las personas quieren sea potenciado requiere esclarecer su relación con los de actitud, motivo y necesidad, estableciendo un vínculo íntimo entre el sujeto y el valor con una carga afectiva para brindar motivación suficiente al momento de tomar una decisión. Con lo cual el operador contará con todas las herramientas bridadas por la investigación para especializar su profesión aún más con técnicas de persuasión enfocadas a los valores intangibles y el vínculo con el usuario.

Por eso el intangible de la especialización es importante en la investigación, los nuevos conocimientos pretenden facilitar e instruir con mayor contundencia y claridad a los operadores de los MSC con la finalidad de incrementar sus conocimientos y dar una respuesta efectiva a las necesidades de los distintos segmentos, formando cada vez más la profesión de los MSC.

\section{EL POSICIONAMIENTO COMO RESULTADO DE LOS VALORES INTANGIBLES}

Se entiende por posicionamiento la descripción comparada de la percepción del consumidor sobre un servicio entre las demás ofertas en el mercado (Ampuero \& Vila, 2006, pág. 102). Un consumidor de la mediación se enfrenta a las distintas ofertas para resolver un conflicto, estas ofertas se dividen en tres macro opciones: 1. Hacer uso de la vía judicial; 2 . Hacer uso de un método alterno de solución de conflictos, como la mediación; y 3 . Hacer uso de procesos de negociación manejados por abogados o expertos.

En el recorrido teórico hasta el momento se ha planteado la relevancia y relación existente entre los valores intangibles y la ex- 
pectativa del servicio a recibir. Este evento toma lugar en un proceso mental, denominado percepción, en el cual un individuo brinda sentido y coherencia a su entorno y su realidad (Armstrong \& Kotler, 2013, p. 82), seleccionando los valores intangibles, organizando los criterios de valor para el consumidor e interpretándolos según apreciación de valor.

Según la teoría del comportamiento del consumidor (Schiffman \& Kanuk, 2010, p. 161) la selección hace referencia a la capacidad del consumidor para escoger elementos, evento racional o impulsivo, percibidos y considerados como de interés. Aplicado en la mediación este proceso se encarga de identificar de entre todos los elementos del entorno los valores intangibles de la mediación, con el objeto de generar una diferenciación positiva en el consumidor.

Esta diferencia positiva puede verse reflejada en la competitividad entre una mediación y cualquier otra oferta de servicio, ya que los procesos innovadores de la mediación ajustados a las necesidades de los consumidores y el empoderamiento les permite tener control sobre sus propias decisiones para resolver el conflicto.
Si el mensaje es adecuadamente transmitido, el consumidor podrá reconocer en el proceso de selección estos valores intangibles, los cuales serán organizados en el segundo proceso de la percepción. En este momento el consumidor de la mediación divide los estímulos indeseables, representados por la información innecesaria que le rodea, y los estímulos de interés, representados por los valores intangibles que se alinean con sus intereses (Vargas Melgarejo, 1994, p. 47).

En la tercera etapa de la percepción se encuentra la interpretación, siendo el resultado de las dos etapas previas. Es decir, el consumidor percibe un resultado del proceso de la experiencia vívida, situación que le brinda el conocimiento suficiente para poder comparar con la expectativa generada previo al consumo de la mediación. De este resultado se pueden encontrar tres estadios con relación al grado de satisfacción obtenido: insatisfacción, satisfacción y superación de satisfacción.

Kotler y Keller (2012, p. 128) definen a la satisfacción, desde una perspectiva del marketing, como la diferencia entre la expectativa y la experiencia. Utilizando esta ecuación, se presenta a continuación un cuadro descriptivo sobre los distintos resultados de la satisfacción del consumidor de la mediación.

\section{Tabla 1. Resultado de la satisfacción}

\begin{tabular}{|c|l|c|c|}
\hline Medición & Grado de satisfacción & \multicolumn{1}{|c|}{ Posible resultado } & Posicionamiento \\
\hline Expectativa & No supera expectativa & $\begin{array}{l}\text { No piensa en utilizar de } \\
\text { nuevo el servicio }\end{array}$ & Mal posicionamiento \\
\hline
\end{tabular}




\begin{tabular}{|l|l|l|l|}
\hline Expectativa & Iguala expectativa & $\begin{array}{l}\text { Posiblemente vuelva a usar el } \\
\text { servicio }\end{array}$ & $\begin{array}{l}\text { Posicionamiento } \\
\text { reconocido }\end{array}$ \\
\hline Expectativa & Supera expectativa & $\begin{array}{l}\text { Repite el servicio y recomien- } \\
\text { da }\end{array}$ & $\begin{array}{l}\text { Posicionado como la } \\
\text { mejor opción }\end{array}$ \\
\hline
\end{tabular}

Fuente: Basado en Kotler y Keller (2012, p. 128).

Como se puede observar el consumidor de una mediación tiene tres posibles resultados con relación a su satisfacción. La primera obteniendo un resultado no adecuado para ninguna parte de la mediación, este resultado desencadena el desuso de la mediación como método de solución de conflicto, debido a que ningún valor intangible fue reconocido en la percepción del consumidor.

El segundo posible resultado presenta una mejora para los operadores de la mediación, demuestra que es posible marcar en la mente del consumidor a la mediación como un método que le genera valor. Abriendo la puerta para repetir la mediación cuando se encuentre en otro conflicto similar al que anteriormente tuvo, con la lógica de esperar un resultado similar. Empero, los ciudadanos no se encuentran tan frecuentemente en conflictos, es decir, no esperan cursar nuevamente un procedimiento como este, o al menos no lo desean.

El tercer posible resultado presenta un posicionamiento ideal para los servicios, ese estado en la mente del consumidor donde el servicio es la primera opción, se obtiene del resultado de superar las expectativas; al igual al caso anterior los consumidores de la mediación no esperan volver a pasar por un conflicto, el evento de haber superado las expectativas va a permitir la expansión de la mediación en la sociedad por medio de recomendaciones a sus familiares, amigos, vecinos o conocidos. Siendo este último el objetivo por buscar de los valores intangibles de la mediación.

\section{CONCLUSIONES}

El presente artículo abordó los valores intangibles de la mediación desde la perspectiva teórica del comportamiento del consumidor del marketing, haciendo una descripción teórica conceptual del valor intangible, su aplicabilidad en el mundo de los negocios de servicios como lo es la mediación. Se describió cómo el consumidor percibe preceptos considerados valiosos y otros desechables, dándole relevancia al tema de la percepción.

El consumidor actualmente no puede reconocer todos los estímulos que brinda una oferta de valor en los servicios, a causa del exceso de información existente en el entorno. Entre tantas ofertas de servicios para una misma demanda, prevalecen únicamente aquellas que pueden enfatizar los beneficios de una manera distintiva y ajustada a la necesidad del consumidor, por medio de los valores intangibles. 
Los valores intangibles de la medición presentados en este trabajo se refieren a factores de relevancia para el posicionamiento desde la perspectiva económica y administrativa en el mercado, como generadores de valor constante: la competitividad, la innovación, la modernidad, el empoderamiento y la especialización. Intangibles generadores de valor que aportan al posicionamiento de los consumidores de la mediación.

Estos valores intangibles de la mediación en su conjunto ofrecen mejoras estructurales para los procesos de mediación, dando como resultado sistemas diferenciados con mejores grados de satisfacción, diseñados para influenciar a los consumidores en reconocer estos valores intangibles y posicionarla como la opción número uno para una necesidad.

Es así como, los valores intangibles han sido factores de mejoras y desarrollo en los servicios, innovando en las formas de brindar soluciones a las necesidades de los individuos. Al mismo tiempo el esfuerzo desplegado en la implementación de los valores intangibles resulta en servicios de mejor calidad, mayor eficiencia y eficacia al estudiar las oportunidades de mejora existente en los consumidores.

La innovación va de la mano con procesos de modernización necesaria que se ajusta a las necesidades de los consumidores, al igual que los productos, los servicios sino se adecúan a la demanda existente, la tendencia a extinguirse se incrementa considerablemente. Por esta razón los procesos de mejora continua son impulsados por el valor intangible que facilita la adaptación de los servicios a los intereses del consumidor y su comportamiento.

Como bien se esbozó anteriormente los procesos de modernización e innovación van de la mano con los cambios sociales que se reflejan en el comportamiento de los consumidores. Estos cambios conductuales del consumidor han llegado al punto de ser ellos mismos quienes decidan y controlen que sucede con sus decisiones abiertamente, transformándose en un agregado de valor para la mediación.

Todo lo planteado toma lugar en la percepción del consumidor en tres fases consecutivas que selecciona los valores percibidos por medio de estímulos, para posteriormente ser organizados en forma prioritaria y con base a sus intereses, lo que termina en una interpretación de la experiencia del servicio, ubicándolo mentalmente ya sea: en no volver a utilizarlo, volver a utilizarlo y recomendación.

\section{TRABAJOS CITADOS}

Ampuero, O., \& y Vila, N. (1 de Febrero 2006). Consumer perceptions of product packaging. Journal of Consumer Marketing. 23 (2), 100-112. Recuperado en: https://www. researchgate.net/publication/235303347_Consumer_ perception_of_product_packaging

Aranguren Álvarez, W. (2015). Modernidad y desarrollo humano: elementos discursivos y controversiales. Negotium Revista Científica Electrónica de Ciencias Gerenciales, 11 (32) pp. 52-67. Recuperado en: https://www. redalyc.org/pdf/782/78246590003.pdf

Armstrong, G., \& y Kotler, P. (2013). Fundamentos de Marketing (11 ed.). México, D.F.: Pearson. 
Ávalos Rodríguez, I. (2017). Bioaprendizaje en la educación virtual. Una reflexión a partir del significado del aprendizaje. VEC Revista Virfualidad, Educación y Ciencia, 8 (15), pp. 147-161. Recuperado en: https://revistas. unc.edu.ar/index.php/vesc/issue/view/Vol\%208\%20 -\%20N\%C3\%BAm\%2015

Caicedo Muñoz, S. C., \& y Solarte-Pazos L. (2015). Empoderamiento de mujeres de una ONG colombiana. Un estudio de caso simple. Revista de administração pública (Rio de Janeiro), 49 (6) pp. 1597-1618. Recuperado en: https://www.scielo.br/j/rap/a/x3yqcHQXY8XDJQRNGcgGWqw/?lang=es

Domínguez-Cruz, J. J., Pereyra-Rodríguez, J. J., \& Ruiz-Villaverde, R. (2018). Empoderamiento y Dermatología. Revista Actas Dermo-Sifiliográficas, 109 (2) pp. 133-139. Recuperado en: https://www.sciencedirect.com/science/article/abs/pii/S1578219017304201

Gil Saura, I., \& González Gallarza, M. (2008). La investigación en valor percibido desde el marketing. INNOVAR. Revista de Ciencias Administrativas y Sociales 18 (31) pp. 19-18. Recuperado en: https://revistas.unal.edu.co/ index.php/innovar/article/view/19766/20870

González Serra, D. J. (2000). Los valores y su formacion: una interpretación psicológica. Revista Cubana de Psicología, 17 (3) pp. 307-311. Recuperado en: http://pepsic.bvsalud.org/pdf/rcp/v17n3/13.pdf

González Ulloa Aguirre, P. A. (2014). Innovación y economía del conocimiento. ¿Qé hay que aprender para México y cuál es el papel de las élites? Revista Enfoques: Ciencia Politíca y Administración Pública, 12 (20) pp. 133-156. Recuperado en: http://www.revistaenfoques.cl/index.php/ revista-uno/article/view/15

Gorjón Gómez, F. J. (2017). Mediación, su valor intangible y efectos operativos. Ciudad de México: Tirant Lo Blanch.

Kotler, P., \& Keller, K. (2012). Dirección de Marketing, 14va ed. México, D.F.: Pearson Eduacación.

López Ruiz, V. R., Nevado Peña, D., \& Baños Torres, J. (2008). Indicador sintético de capital intelectual: humano y estructural. Un factor de competitividad. EURE Revista Latinoamericana de Estudios Urbano Regionales 34 (101), pp. 45-70. Recuperado en: http://www.eure.cl/index. php/eure/article/view/1335

Martínez Corts, I., Munduate Jaca, L., \& Medina Díaz, F. J. (2008). Efectividad de los patrones de influencia. Revista Psicothema, 20, (3) pp. 369-375. Recuperado en: http://www.psicothema.com/pdf/3494.pdf

Moraleda Martínez, A. (2004). La innovación, clave para la competitividad empresarial. Universia Business Review, (1) pp. 128-136. Recuperado en: https://www.redalyc. org/pdf/433/43300112.pdf

Ortega y Gasset, J. (2004). Introducción a una estimativa. ¿Qué son los valores? Revista de Occidente, 3-41. Obras escogidas tomo 6. Madrid: Ediciones Encuentro. Recuperado en: https://www.salud.gob.ec/wp-content/ uploads/2016/12/2.2.-Ortega-Que-son-los-valores.pdf

Rivera Godoy, J. A. (2012). La incidencia de la innovación sobre la creación de valor: propuesta de un modelo desde la perspectiva financiera. Revista Facultad de Ciencias Económicas: Investigación y Reflexión, XX (2) pp. 175-187. Recuperado en: https://revistas.unimilitar. edu.co/index.php/rfce/article/view/2171/1773

Romero Hernández, J. L. (2002). ¿Qué es la modernidad? Revista Planeación y Evaluación Educativa. (24), pp. 1526. Recuperado en: https://publicaciones-aragon.unam. $\mathrm{mx} /$ repositorio/planeacion/24.pdf

Sánchez-Vidal, A. (2017). Empoderamiento, liberación y desarrollo humano. Psychosocial Intervention, 26 (3) pp. 155-163. Recuperado en: https://journals.copmadrid. org/pi/archivos/articulo20171222175149.pdf

Schiffman, L. G., \& y Kanuk, L. L. (2010). Comportamiento del Consumidor. México,D.F.: Pearson.

Torres Citraro, L. (2016). Innovación, portal hacia el futuro. Revista la Propiedad Inmaterial, (21), pp. 111-137. Recuperado en: https://revistas.uexternado.edu.co/index. php/propin/article/view/4604/5292

Unger Rubin, K., Flores Peregrina, D., \& y Ibarra-Olivo, J. E. (2014). Productividad y capital humano. Fuentes complementarias de la competitividad en los estados en México. EL TRIMESTRE ECONÓMICO, LXXXI (4) (324) pp. 909-941. Recuperado en: https://www.eltrimestree- 
conomico.com.mx/index.php/te/article/view/134

Urbina-Medina, H., Noguera Brizuela, D., Levy Mizhary, J., Carrizo Chuecos, J.T., \& y Betancourt A. (2016). Comunicación efectiva y ética en casos de epidemias y pandemias. Archivos venezolanos de puericultura y pediatría, 79 (4) pp. 113-117. Recuperado en: https://www.redalyc. org/pdf/3679/367949933002.pdf

Vargas Melgarejo, L. M. (1994). Sobre el concepto de percepción. Revista Alteridades, (8) pp. 47-53. Recuperado en: https://alteridades.izt.uam.mx/index.php/Alte/article/view/588/586

$-$

\section{Vanny Maltez Navarro}

Abogado, investigador, empresario y catedrático, Actual doctorando en Métodos Alternos de Solución de Conflictos en la Universidad Autónoma de Nuevo León. Máster en Dirección Empresarial enfocado en Dirección Financiera, Mediador y árbitro certificado en Nicaragua, Licenciado en Derecho y Administración de Empresas, miembro red CLACSO y parte del gremio investigador de la ciencia de los MSC. 